\title{
Metabolic Engineering of Escherichia coli for Production of Polyhydroxyalkanoates with Hydroxyvaleric Acid Derived from Levulinic Acid
}

\author{
Doyun Kim ${ }^{1}$ and Sung Kuk Lee $\mathrm{L}^{1,2,3 *}$ \\ ${ }^{1}$ Department of Biomedical Engineering, Ulsan National Institute of Science and Technology (UNIST), Ulsan 44919, \\ Republic of Korea \\ ${ }^{2}$ Department of Chemical Engineering, Ulsan National Institute of Science and Technology (UNIST), Ulsan 44919, \\ Republic of Korea \\ ${ }^{3}$ Department of Energy Engineering, Ulsan National Institute of Science and Technology (UNIST), Ulsan 44919, \\ Republic of Korea
}

\begin{abstract}
Polyhydroxyalkanoates (PHAs) are emerging as alternatives to plastics by replacing fossil fuels with renewable raw substrates. Herein, we present the construction of engineered Escherichia coli strains to produce short-chain-length PHAs (scl-PHAs), including the monomers 4-hydroxyvalerate (4HV) and 3-hydroxyvalerate (3HV) produced from levulinic acid (LA). First, an E. coli strain expressing genes (IvaEDABC) from the LA metabolic pathway of Pseudomonas putida KT2440 was constructed to generate 4HV-CoA and 3HV-CoA. Second, both PhaAB enzymes from Cupriavidus necator $\mathrm{H} 16$ were expressed to supply 3-hydroxybutyrate (3HB)-CoA from acetyl-CoA. Finally, PHA synthase ( PhaC $_{\mathrm{cv}}$ ) from Chromobacterium violaceum was introduced for the subsequent polymerization of these three monomers. The resulting E. coli strains produced four PHAs (w/w\% of dry cell weight): $9.1 \mathrm{wt} \%$
\end{abstract} P(4HV), 1.7 wt\% P(3HV-co-4HV), 24.2 wt\% P(3HB-co-4HV), and 35.6 wt\% P(3HB-co-3HV-co-4HV).

Keywords: Levulinic acid, Escherichia coli, short-chain-length polyhydroxyalkanoates (scl-PHAs)

Received: August 16, 2021 Accepted: October 12, 202

First published online: October 14, 2021

* Corresponding author Phone: +82-52-217-2514 Fax: +82-52-217-3009 E-mail: sklee@unist.ac.kr

pISSN 1017-7825 eISSN 1738-8872

Copyright $(\subset 2022$ by the authors. Licensee KMB. This article is an open access article distributed under the terms and condition of the Creative Commons Attribution (CC BY) license.

\section{Introduction}

Since 2019, the world has been dealing with the effects of the coronavirus pandemic (COVID-19) [1], which has caused wide-ranging environmental and health issues since it was officially classified as a global problem in 2020. One of the serious environmental impacts of the pandemic has been the sudden increase in demand for plastic products known as personal protective equipment (PPE) [2].

Bio-based plastics have emerged as a sustainable alternative to conventional plastics, and can help replace fossil fuels with renewable resources in the short term [3]. The use of bio-based plastics can reduce their carbon footprint, and biodegradable plastics increase the efficiency of plastic recycling and waste management, thereby lessening the environmental impact of plastic waste $[4,5]$. Polyhydroxyalkanoates (PHAs) are highly appealing polymers derived from biomass and used as biodegradable plastics [6]. Among them, poly-3-hydroxybutyrate $[\mathrm{P}(3 \mathrm{HB})]$ has been researched most.

$\mathrm{P}(3 \mathrm{HB})$ is a 3-hydroxybutyrate (3HB)-based short-chain-length PHA (scl-PHA) with physical properties similar to those of petroleum-based plastics, including polypropylene and polystyrene [7]. However, $\mathrm{P}(3 \mathrm{HB})$ is difficult to manufacture because of its high brittleness and high melting point, both limiting factors in its production and application $[8,9]$. Glass and melting transition temperatures are important parameters related to the application of PHA [10]. To solve this problem, studies have been conducted to control PHA composition, and it has been confirmed that the properties of PHA can be improved by altering its composition [11]. Incorporating 4-hydroxyvalerate (4HV) or 3-hydroxyvalerate (3HV) monomers into PHAs has been shown to lower the melting point and improve flexibility without affecting the plastic decomposition efficiency [11-13]. However, the production of such copolymer PHAs has been limited owing to the high toxicity of petroleum-derived precursors like $\gamma$-valerolactone (GVL), valeric acid, or propionate, and the high cost of biological production of $4 \mathrm{HV}$ and $3 \mathrm{HV}$ monomers $[8,14,15]$.

Renewable carbon sources are economically reasonable and have excellent potential as feedstock for industrial PHA production [10]. Levulinic acid (LA) is considered a $3 \mathrm{HV}$ and $4 \mathrm{HV}$ precursor for PHA production [16-18]. In addition, LA can be produced on industrial scale from cellulosic biomass at a cost as low as $\$ 0.04-\$ 0.10 / \mathrm{lb}[19]$. An engineered Pseudomonas putida strain showed potential for effective PHA production from LA using its LA 
metabolic pathway [18]. However, use of this Pseudomonas strain is hindered by incomplete knowledge of genetic information and non-availability of genetic manipulation tools when using low-cost carbon sources.

Biological PHA production studies should aim to improve the cost and efficiency of the process. Most natural microorganisms can easily synthesize PHA from various monomers, but only on laboratory-scale and from structurally related precursors [20]. PHA biosynthesis in hosts that do not naturally produce PHA may improve the quality and quantity of PHA [21]. In addition, the PHA synthesis pathway can also be modified to extend the range of products and control the monomer content [21]. Escherichia coli generally does not produce PHA, but its short doubling time and the comprehensive knowledge of its molecular genetics and physiology have made E. coli a pioneering organism in the study of PHA biosynthesis [22-25].

In this study, we investigated the biosynthesis of different forms of PHA in E. coli engineered with the LA catabolic pathway (lva pathway) derived from P. putida KT2440 [26]. The present study also proposes an alternative method to effectively supply HV monomers for PHA synthesis compared to using petroleum-derived precursors (GVL, valeric acid, or propionate).

\section{Materials and Methods}

\section{Construction of Bacterial Strains and Plasmids}

Table 1 lists the strains and plasmids used in this research. E. coli DH10B was used for all molecular cloning experiments. It was used as the parent strain of E. coli MG1655 developed for PHA production. P. putida KT2440 (DSMZ 6125) was procured from the German Collection of Microorganisms and Cell Cultures GmbH (DSMZ, Germany). Cupriavidus necator H16 (KCTC 22469) and Chromobacterium violaceum (KCTC 2897) were purchased from the Korean Collection for Type Cultures (KCTC, Korea).

Q5 High-Fidelity DNA polymerase, Taq DNA ligase, T4 polynucleotide kinase, and T5 exonuclease were procured from New England Biolabs (USA) and used for PCR and plasmid construction. All plasmids used in this study were constructed using the Biobrick plasmid [27]. To create pBbB6a-lvaED, pBbE6k-lvaABC, pBbA2cphaC, and pBbA2c-phaCAB plasmids, the genes lvaEDABC of P.putida KT2440, phaC of C. violaceum, and phaAB of $C$. necator were amplified individually and cloned into each plasmid [28]. The RBS sequence was newly synthesized and applied to each gene using the Salis Lab RBS calculator. (Version 2.1) [29]. The plasmid was then transformed into E. coli using a MicroPulser electroporator (Bio-Rad, USA).

\section{Media and Cultivation Conditions}

LA solution (Sigma-Aldrich, USA) was neutralized to $\mathrm{pH} 7$ with $\mathrm{NaOH}$ and used for cultivation. For the gas chromatography-mass spectrometry (GC-MS) analysis, methyl benzoate (Acros Organics, USA), GVL, and PHA polymer granules ( $88 \mathrm{~mol} \% 3 \mathrm{HB}, 12 \mathrm{~mol} \% 3 \mathrm{HV}$ ) (Sigma-Aldrich) were used. GVL was saponified with $\mathrm{NaOH}$ to prepare 4-hydroxyvaleric acid for GC-MS [30].

The recombinant $E$. coli strains were inoculated in $5 \mathrm{ml} \mathrm{lysogeny} \mathrm{broth} \mathrm{(LB)}(5 \mathrm{~g} / \mathrm{l}$ yeast extract, $10 \mathrm{~g} / \mathrm{l}$ tryptone, and $10 \mathrm{~g} / \mathrm{l} \mathrm{NaCl}$ ) and cultured at $37^{\circ} \mathrm{C}$ for $10 \mathrm{~h}$ with $200 \mathrm{rpm}$ shaking. For PHA production, the grown seed culture was transferred (1:40) into $40 \mathrm{ml} \mathrm{MR}$ medium ( $\mathrm{pH}$ 7) containing: $4 \mathrm{~g} / \mathrm{l}\left(\mathrm{NH}_{4}\right)_{2} \mathrm{HPO}_{4}, 6.67 \mathrm{~g} / \mathrm{K} \mathrm{KH}_{2} \mathrm{PO}_{4}, 0.8 \mathrm{~g} / \mathrm{l}$ citric

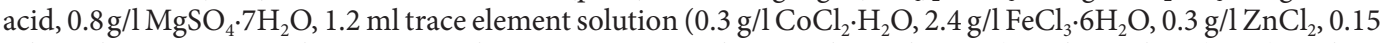
$\mathrm{g} / \mathrm{l} \mathrm{CuCl} 2 \cdot 2 \mathrm{H}_{2} \mathrm{O}, 0.075 \mathrm{~g} / \mathrm{H} \mathrm{H}_{3} \mathrm{BO}_{3}, 0.3 \mathrm{~g} / \mathrm{l} \mathrm{Na}_{2} \mathrm{MO}_{4} \cdot 2 \mathrm{H}_{2} \mathrm{O}$, and $0.495 \mathrm{~g} / \mathrm{l} \mathrm{MnCl}{ }_{2} \cdot 4 \mathrm{H}_{2} \mathrm{O}$ ), and $2.3 \mathrm{~g} / \mathrm{l} \mathrm{LA}(\mathrm{pH} 7)$, with 15 $\mathrm{g} / \mathrm{l}$ glucose. The bacteria were cultured in a $250-\mathrm{ml}$ shake flask at $30^{\circ} \mathrm{C}$ for $96 \mathrm{~h}$ with $200 \mathrm{rpm}$ shaking. When the optical density $\left(\mathrm{OD}_{600}\right)$ reached $0.3,0.1 \mathrm{mM}$ isopropyl-D-1-thiogalactopyranoside (IPTG) and $50 \mathrm{nM}$ tetracycline were added to induce gene expression. The medium was supplemented with ampicillin $(100 \mu \mathrm{g} / \mathrm{l})$, chloramphenicol $(30 \mu \mathrm{g} / \mathrm{l})$, and/or kanamycin $(50 \mu \mathrm{g} / \mathrm{l})$ depending on the resistance marker of the plasmids. A spectrophotometer was used to measure the $\mathrm{OD}_{600}$ (Libra S22; Biochrom, UK).

Table 1. Strains and plasmids.

\begin{tabular}{|c|c|c|}
\hline Strains and plasmids & Genotype and description & Reference \\
\hline \multicolumn{3}{|l|}{ Strains } \\
\hline MG1655 & E. coli K-12 $\mathrm{F}^{-} \lambda^{-} i l v G^{-} r f b-50 r p h-1$ & [41] \\
\hline DH10B & $\begin{array}{l}\mathrm{F}^{-} \text {mcrA } \Delta(\text { mrr-hsdRMS-mcrBC) } \varphi 80 \text { lacZ } \Delta \mathrm{M} 15 \Delta \text { lacX74 recA1 endA1 araD139 } \\
\Delta(\text { ara-leu }) 7697 \text { galU galK } \lambda^{-} r p s L\left(\operatorname{Str}^{R}\right) \text { nupG }\end{array}$ & [42] \\
\hline PHV01 & MG1655 harboring pBbB6a-lvaED, and pBbA2c-phaC & This study \\
\hline PHV11 & MG1655 harboring pBbB6a-lvaED, and pBbE6k-lvaABC, and pBbA2c-phaC & This study \\
\hline PHBV01 & MG1655 harboring pBbB6a-lvaED, and pBbA2c-phaCAB & This study \\
\hline PHBV11 & MG1655 harboring pBbB6a- $l v a E D$, pBbE6k-lvaABC, and pBbA2c-phaCAB & This study \\
\hline \multicolumn{3}{|l|}{ Plasmids } \\
\hline pBbB6a-gfp & pBBR1 origin, carrying $g f p$ under the $\mathrm{P}_{\text {Llaco-1 }}, \mathrm{Amp}^{\mathrm{R}}$ & [27] \\
\hline pBbE6k-rfp & ColE1 origin, carrying $r f p$ under the $\mathrm{P}_{\text {Llaco-1 }}, \mathrm{Km}^{\mathrm{R}}$ & [27] \\
\hline $\mathrm{pBbA} 2 \mathrm{c}-r f p$ & p15A origin, carrying $r f p$ under the $\mathrm{P}_{\text {tetA }}, \mathrm{Cm}^{\mathrm{R}}$ & {$[27]$} \\
\hline pBbB6a-lvaED & pBbB6a-g $f p$ with $\triangle g f p:: l v a E D$ from P. putida KT2440, Amp ${ }^{\mathrm{R}}$ & This study \\
\hline pBbE6k-lvaABC & pBbE6k-rfp with $\triangle r f p:: l v a A B C$ from P. putida KT2440, $\mathrm{Km}^{\mathrm{R}}$ & This study \\
\hline pBbA2c-phaC & pBbA2c-rfp with $\Delta r f p:: p h a C$ from C. violaceum, $\mathrm{Cm}^{\mathrm{R}}$ & This study \\
\hline pBbA2c-phaCAB & pBbA2c-rfp with $\triangle r f p::$ phaCAB from C. violaceum, and $C$. necator $\mathrm{H} 16, \mathrm{Cm}^{\mathrm{R}}$ & This study \\
\hline
\end{tabular}


PHA Analytical Methods

For the PHA study, $10 \mathrm{ml}$ of culture was centrifuged for $20 \mathrm{~min}$ at $4^{\circ} \mathrm{C}$ at $2,600 \times g$ (Swingout rotor, Combi-514R, Hanil Scientific, Korea). The harvested cells were washed twice with triple-distilled water and the pellet was frozen at $-80^{\circ} \mathrm{C}$ using a $2.5-\mathrm{L}$ benchtop freeze dryer (Labconco, USA). PHA content and composition were analyzed by GC-MS using the following methanolysis method [31]: the dried cells were treated with a mixture of $1 \mathrm{ml}$ chloroform, $1 \mathrm{ml}$ of $15 \%(\mathrm{v} / \mathrm{v})$ methanol, and $85 \%(\mathrm{v} / \mathrm{v})$ sulfuric acid in $8 \mathrm{ml}$ glass vials (WH224704, Wheaton, USA), and reacted in a dry bath (MaXtable H10, DAIHAN, Korea) at $100^{\circ} \mathrm{C}$ for $3 \mathrm{~h}$. A polytetrafluoroethylenelined cap was used to close the vials (WH240409, Wheaton, Millville, New Jersey, USA). The vials were cooled to room temperature after the reaction, and $1 \mathrm{ml}$ of a $0.5 \%(\mathrm{v} / \mathrm{v})$ methyl benzoate-chloroform solution and $1 \mathrm{ml}$ triple distilled water were applied for phase acceleration and vortexed for $30 \mathrm{~s}$. The phase separation was performed at room temperature $\left(20 \sim 22^{\circ} \mathrm{C}\right)$ for $3 \mathrm{~h}$. The sample $(500 \mu \mathrm{l})$ obtained in the organic solvent layer was used for GCMS analysis.

The analysis was performed on a 7890 B GC-MS (Agilent Technologies) system equipped with an Agilent J\&W CycloSil-B column (113-6632). Helium was used at a flow rate of $1.5 \mathrm{ml} / \mathrm{min}$, and $2 \mu \mathrm{l}$ of the sample was injected. From a starting temperature of $60^{\circ} \mathrm{C}$, the oven temperature was raised at the rate of $10^{\circ} \mathrm{C} / \mathrm{min}$ for $7 \mathrm{~min}$, and then increased at the rate of $30^{\circ} \mathrm{C} / \mathrm{min}$ until reaching $250^{\circ} \mathrm{C}$. All experiments were performed in triplicate.

\section{Results and Discussion}

\section{Construction of Engineered E. coli for PHA Production}

$\mathrm{P}(3 \mathrm{HB})$ is the most widespread and best-characterized form of PHA and is produced by various bacterial species such as Cupriavidus necator [1], Alcaligenes latus [2], Bacillus spp. [4], Azotobacter vinelandii [3], and Pseudomonas sp. [4]. The incorporation of other monomer units into the $3 \mathrm{HB}$ polymer chains can result in copolymers with improved properties. Poly(3-hydroxybutyrate-co-3-hydroxyvalerate) is among the most popular copolymers, containing $3 \mathrm{HV}$ produced from propionic acid, propanol, valeric acid, pentanol, or heptanoic acid.

Recently, a metabolic pathway in the lva operon that can synthesize $4 \mathrm{HV}-\mathrm{CoA}$ and $3 \mathrm{HV}-\mathrm{CoA}$ from LA, which can be cost-effectively produced from renewable cellulosic biomass, was identified in P. putida and characterized [26]. To synthesize HV monomer units from LA in E. coli, P. putida lvaED and $l v a A B C$ were cloned into pBbE6a$g f p$ and pBbE6k-rfp by replacing $g f p$ and $r f p$, respectively, generating pBbB6a-lvaED and pBbE6k-lvaABC plasmids. pBbB6a-lvaED was introduced into E. coli to produce $4 \mathrm{HV}-\mathrm{CoA}$ and $\mathrm{pBbE} 6 \mathrm{k}-\mathrm{lva} A B C$ to produce $3 \mathrm{HV}$ CoA (Fig. 1).

C. necator $\mathrm{H} 16$ is a model microorganism for $\mathrm{PHB}$ production using the phaABC operon. To synthesize $3 \mathrm{HB}$ monomer units from glucose in $E$. coli, the $p h a A B$ genes encoding acetyl-CoA reductase and acetyl-CoA acetyltransferase from C. necator were cloned into pBbA2c-rfp together with PHA synthase $\left(\mathrm{PhaC}_{\mathrm{Cv}}\right)$ from $C$. violaceum under the tetracycline-inducible promoter $\left(\right.$ tet $\left.R-\mathrm{P}_{\text {tet }}\right)$ to generate $\mathrm{pBbA2c}-\mathrm{phaCAB}$. To produce $\mathrm{PHAs}$ with HVs in E. coli, PHA synthase $\left(\mathrm{PhaC}_{\mathrm{CV}}\right)$ from $C$. violaceum was expressed on the $\mathrm{pBbA2}$ plasmid under the tetracycline-inducible promoter $\left(t e t R-\mathrm{P}_{\mathrm{tetA}}\right)$ with and without $C$. necator phaAB, generating pBbA2c-phaCAB and pBbA2c-phaC plasmids, respectively.

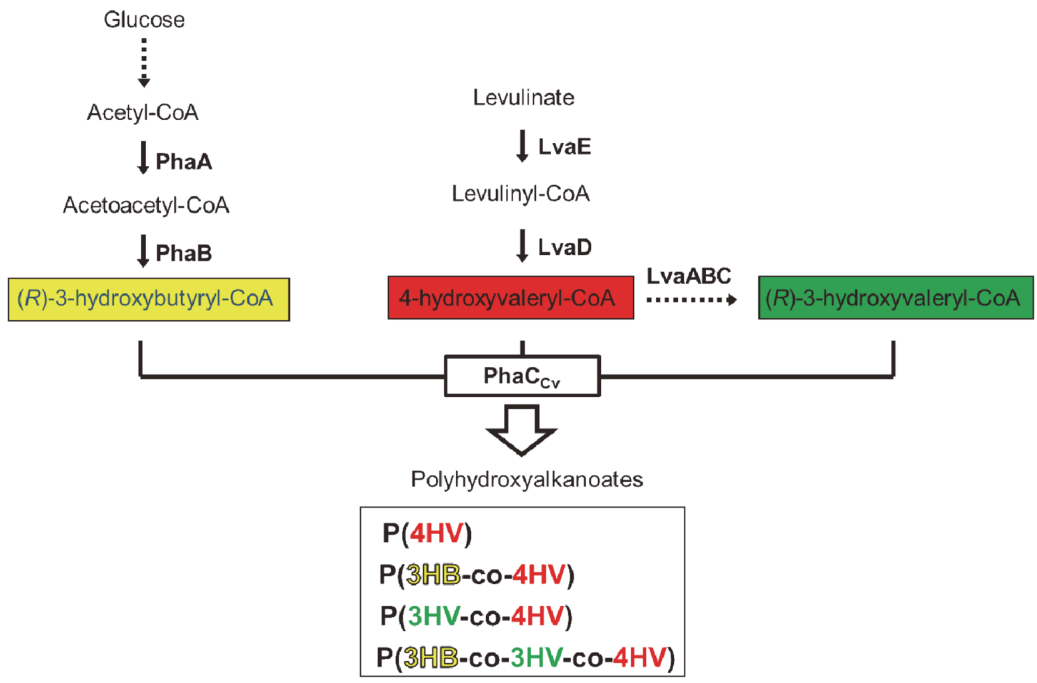

Fig. 1. Metabolic pathways involved in the production of scl-PHA in this study. Enzymes: PhaA, acetyl-CoA acetyltransferase from Cupriavidus necator H16; PhaB, acetoacetyl-CoA reductase from C. necator H16; LvaA, 4hydroxypentanoyl-CoA kinase; LvaB, 4-hydroxypentanoyl-CoA kinase; LvaC, 4-(phosphooxy)pentanoyl-CoA phosphatase/ mutase; LvaD, 4-oxopentanoyl-CoA 4-dehydrogenase; LvaE, short-chain acyl-CoA synthetase; $\mathrm{PhaC}_{\mathrm{C} w}$ poly(R)-3hydroxyalkanoate polymerase from Chromobacterium violaceum. 
Table 2. PHA content in the cell biomass (wt\%), monomer molar ratio (mol\%) and dried cell weight $(\mathrm{g} / \mathrm{l})$ of strains.

\begin{tabular}{lcccc}
\hline & PHV01 & PHV11 & PHBV01 & PHBV11 \\
\hline 3HB (mol\%) & $\operatorname{Tr}^{\mathrm{a}}$ & $\operatorname{Tr}^{\mathrm{a}}$ & $85.3 \pm 1.0$ & $87.9 \pm 0.8$ \\
3HV (mol\%) & $\mathrm{ND}^{\mathrm{b}}$ & $82.4 \pm 0.9$ & $\mathrm{ND}^{\mathrm{b}}$ & $5.7 \pm 0.2$ \\
4HV (mol\%) & $98.7 \pm 0.1$ & $\operatorname{Tr}^{\mathrm{a}}$ & $14.6 \pm 1.0$ & $6.4 \pm 0.6$ \\
CDM (g/l) & $1.6 \pm 0.0$ & $1.6 \pm 0.1$ & $1.8 \pm 0.0$ & $1.4 \pm 0.0$ \\
PHA content (wt\%) & $9.1 \pm 0.7$ & $1.7 \pm 0.1$ & $24.2 \pm 2.8$ & $35.6 \pm 2.2$ \\
\hline
\end{tabular}

${ }^{a} \mathrm{Tr}$, Detected in trace quantity $(<0.5 \%, \mathrm{w} / \mathrm{w})$

${ }^{\mathrm{b}} \mathrm{ND}$, Not detectable

Poly-4-Hydroxyvalerate (p(4HV)) Production

Although E. coli does not naturally produce $\mathrm{P}(3 \mathrm{HB})$, recombinant E. coli strains harboring the C. necator PHA biosynthesis genes have been used to efficiently produce $\mathrm{P}(3 \mathrm{HB})$ mostly from glucose [32].

To investigate the possibility of high $\mathrm{P}(4 \mathrm{HV})$ production from $\mathrm{LA}$, a promising platform chemical that can be obtained from biomass, we constructed E. coli MG1655 harboring pBbB6a-lvaED and pBbA2c-phaC. In this system, only two enzymatic reactions are involved in the production of $4 \mathrm{HV}-\mathrm{CoA}$ from LA, which is then polymerized by $\mathrm{PhaC}_{\mathrm{Cv}}$ to form $\mathrm{P}(4 \mathrm{HV})$, compared with the 12 enzymatic steps required for $3 \mathrm{HB}-\mathrm{CoA}$ synthesis from glucose for $\mathrm{P}(3 \mathrm{HB})$ production [33]. Approximately $1.6 \mathrm{~g} / \mathrm{l}$ dry cell weight (DCW) with $9.0 \mathrm{wt} \% \mathrm{P}(4 \mathrm{HV})$ content was obtained from $2.3 \mathrm{~g} / \mathrm{l} \mathrm{LA}$ after $96 \mathrm{~h}$ cultivation of the PHV01 strain (Table 2). Even without the expression of PhaAB, trace amounts of $3 \mathrm{HB}$ (approximately $0.1 \mathrm{wt} \%$ ) were incorporated into the PHA backbone. This might be due to 3-hydroxyacyl-CoA epimerase (FadB, FadJ) activity in the cell that converts (S)-3hydroxybutyl-CoA released during beta-oxidation to (R)-3-hydroxybutyryl-CoA [34].

In previous $\mathrm{P}(4 \mathrm{HV})$ production studies, $P$. putida KT2440 with lvaAB deletion accumulated only a small amount ( $2 \mathrm{wt} \%$ cell content) of the $\mathrm{P}(4 \mathrm{HV})$ homopolymer from $7.5 \mathrm{~g} / \mathrm{LAA}[18]$ and an engineered $E$. coli strain also produced a small amount (1 wt $\%)$ of $\mathrm{P}(4 \mathrm{HV})$ from $4 \mathrm{HV}$ as a substrate [35]. In this study, a higher (9.0 wt\%) $\mathrm{P}(4 \mathrm{HV})$ content was produced than those in previous studies; nevertheless, this amount is still low compared with the $\mathrm{P}(3 \mathrm{HB})$ production of up to $80 \mathrm{wt} \%$ (Fig. 2) [36]. Considering a previous study that showed production of $100 \mathrm{~g} / \mathrm{l}$ and $4.2 \mathrm{~g} / \mathrm{l} / \mathrm{h}$ of $4 \mathrm{HV}$ from LA in E. coli MG1655 [37], the low production of $\mathrm{P}(4 \mathrm{HV})$ in the present study

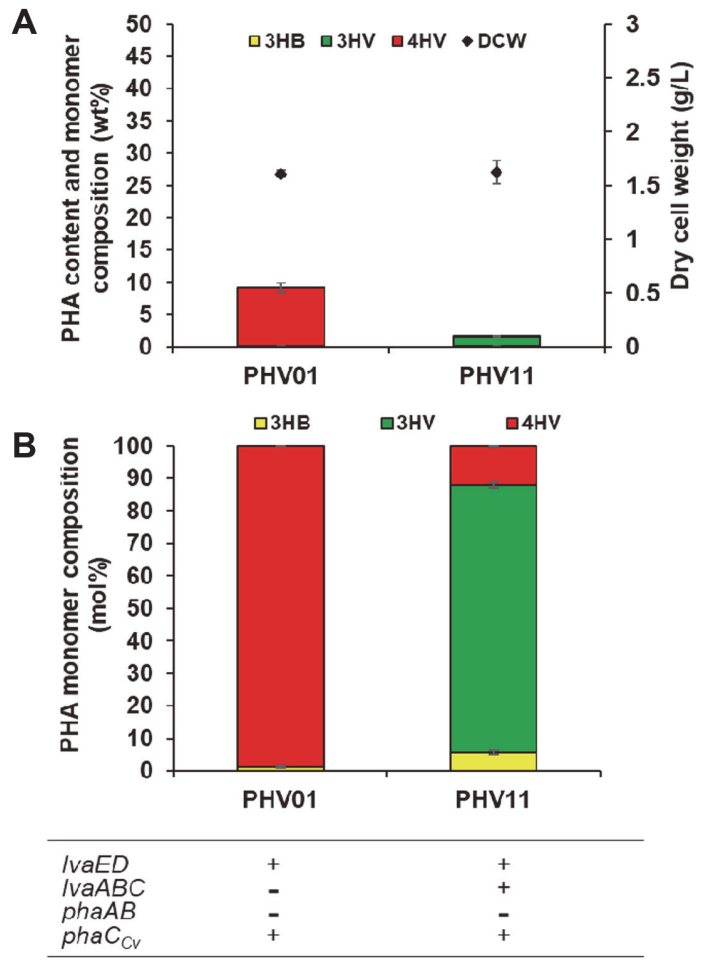

Fig. 2. Cellular PHA content (wt\%), dry cell weight (g/l) (A) and PHA monomer composition (mol\%) (B) in PHV01 strain harboring pBbB6a-lvaED and pBbA2c-phaC, and PHV11 strain harboring pBbB6a-lvaED, pBbE6k-lvaABC and pBbA2c-phaC. Samples were taken and analyzed after $96 \mathrm{~h}$ of cultivation. Yellow, $3 \mathrm{HB}$; red, 4HV; green, $3 \mathrm{HV}$. The table shows the names of plasmid-expressed enzymes. Error bars represent the standard deviations of three independent cultivations. 
may be due to the low activity of the PHA synthase $\mathrm{PhaC}_{\mathrm{CV}}$ for $4 \mathrm{HV}-\mathrm{CoA}$, an unnatural substrate, and because the availability of $4 \mathrm{HV}-\mathrm{CoA}$ for the synthesis of $\mathrm{P}(4 \mathrm{HV})$ was not optimized. This study suggests that enzyme and metabolic engineering must be further explored to increase high $\mathrm{P}(4 \mathrm{HV})$ production.

\section{Poly-3-Hydroxyvalerate-co-4-Hydroxyvalerate [P(3HV-co-4HV)] Production}

$\mathrm{P}(4 \mathrm{HV})$ production in microorganisms was still low $(9.0 \mathrm{wt} \%)$, possibly owing to the low substrate specificity of the PHA synthase for $4 \mathrm{HV}-\mathrm{CoA}$. Because $4 \mathrm{HV}-\mathrm{CoA}$ and $3 \mathrm{HV}-\mathrm{CoA}$ could be generated from LA via the lva operon, we attempted to produce a copolymer. The $\mathrm{PhaC}_{\mathrm{Cv}}$ of $C$. violaceum accumulates polymers comprising predominantly $3 \mathrm{HV}$ [38]. Therefore, the production of $\mathrm{P}(3 \mathrm{HV}-c o-4 \mathrm{HV})$ copolymer was investigated in the PHV11 strain by expressing the polymerase together with LvaED and LvaABC.

After $96 \mathrm{~h}$ of cultivation, a DCW of $1.6 \mathrm{~g} / \mathrm{l}$ and $\mathrm{P}(3 \mathrm{HV}-\mathrm{co}-4 \mathrm{HV})$ copolymer with a molar ratio of $82.4 \mathrm{~mol} \%$ of $3 \mathrm{HV}$ and trace amounts of $4 \mathrm{HV}$ were produced. The highest PHA concentration was determined to be $27.2 \mathrm{mg} / \mathrm{l}$, which corresponds to a PHA content of $1.7 \mathrm{wt} \%$ of DCW. In contrast to the PHV01 expressing only LvaED, the molar proportion of $3 \mathrm{HV}$ in the copolymer rose to $82.4 \mathrm{~mol} \%$ (Fig. 2). In a previous study, a molar ratio of 61.6 $\mathrm{mol} \% 3 \mathrm{HV}$ and $37.8 \mathrm{~mol} \% 4 \mathrm{HV}$ was achieved in P. putida $\mathrm{KT} 2440$ with the same $\mathrm{PhaC}_{\mathrm{Cv}}$ enzyme but no PhaAB expression [18]. P. putida $\mathrm{KT} 2440$ produced $37.4 \mathrm{wt} \% \mathrm{P}(3 \mathrm{HV}-c o-4 \mathrm{HV})$, which was approximately 22 times higher than that produced by the E. coli MG1655 strain.

The PHA monomer composition depends on the amount and proportion of monomers that are fed into the culture medium [39]. In the P. putida KT2440 study, LA was used at a high concentration of $7.5 \mathrm{~g} / \mathrm{l}$ to induce nitrogen limitation [18], whereas $2.3 \mathrm{~g} / \mathrm{l}(20 \mathrm{mM})$ LA was used in this study with E. coli MG1655. P. putida KT2440 probably produced a higher $4 \mathrm{HV}$ monomer proportion than $E$. coli MG1655 owing to the difference in LA concentration. For instance, the molar ratios of $3 \mathrm{HB}, 3 \mathrm{HV}$, and $4 \mathrm{HV}$ were controlled by modulating the substrate concentration in a study on the production of $\mathrm{P}(3 \mathrm{HB}-c 0-4 \mathrm{HV}-c o-3 \mathrm{HV})$ using Cupriavidus sp. USMAA2-4 strain [8]. In another study using the Cupriavidus sp. L7L strain, the monomer proportion of 3HV and cellular PHA content was altered when the quantity of LA in the medium was varied (0.2-1.7\%) [11]. In the present study, only one LA concentration was applied to evaluate the feasibility of PHA production in engineered E. coli. Additional metabolic engineering and LA feeding control can be performed to vary the monomer composition of PHAs. The production of PHA with higher $4 \mathrm{HV}$ proportion by P. putida can also be attributed to the higher rate of LA consumption by $P$. putida than the engineered E. coli PHV11 strain. Improving LA consumption in E. coli through further metabolic engineering can be expected to facilitate higher production of $\mathrm{P}(3 \mathrm{HV}-\mathrm{co}-4 \mathrm{HV})$.

\section{Poly-3-Hydroxybutyrate-co-4-Hydroxyvalerate [P(3HB-co-4HV)] Production}

The inclusion of $4 \mathrm{HV}$ has been proven to improve the physical properties of commercialized PHB [30]. Although petroleum-derived precursors (GVL, valeric acid, or propionate) have been used to supply 4HV, LA, which is easily obtained from biomass, offers economic advantages as a substrate for $4 \mathrm{HV}$ synthesis. We therefore investigated the production of poly(hydroxybutyrate-co-hydroxyvalerate) (PHBV) copolymer from glucose and LA in engineered E. coli PHBV01.

When E. coli PHBV01 expressing LvaED, PhaAB, and $\mathrm{PhaC}_{\mathrm{Cv}}$ was grown in MR minimal medium with LA $(20 \mathrm{mM})$ and glucose $(15 \mathrm{~g} / \mathrm{l}), 1.8 \mathrm{~g} / \mathrm{l} \mathrm{DCW}$ and $435.6 \mathrm{mg} / \mathrm{l}$ PHA titer were obtained after $96 \mathrm{~h}$ cultivation. The cellular PHA content and $4 \mathrm{HV}$ molar ratio were $24.2 \mathrm{wt} \%$ and $14.6 \mathrm{~mol} \%$, respectively (Table 2, Fig. 3). The molar fraction of the non-conventional monomer $4 \mathrm{HV}$ was not very low, even in the presence of the natural monomer $3 \mathrm{HB}$ of PHA synthase. However, the amount of $4 \mathrm{HV}$ decreased from $9.0 \mathrm{wt} \%$ to $3.9 \mathrm{wt} \%$ compared to that of the PHV01 strain without PhaAB expression, indicating that the PHA synthase exhibits higher substrate specificity for 3-hydroxyacyl-CoA (3HB-CoA) than 4-hydroxyacyl-CoA (4HV-CoA).

These results showed that the PHBV copolymer could be produced in a non-natural PHA-producing E. coli strain by introducing heterologous genes. The monomer molar ratio may be varied by controlling the monomer production rate. To increase both PHBV production and $4 \mathrm{HV}$ molar ratio, it is necessary to further engineer PHA synthase to increase its specificity for the non-natural substrate $4 \mathrm{HV}$ over $3 \mathrm{HB}$.

Poly-3-Hydroxybutyrate-co-3-Hydroxyvalerate-co-4-Hydroxyvalerate [P(3HB-co-3HV-co-4HV)] Production Various plastics with specialized properties are required for specific applications. The properties of plastics can be controlled by polymer blending or the production of block copolymers with various monomers. We investigated the production of a random copolymer containing two 3 -hydroxyacids $(3 \mathrm{HB}$ and $3 \mathrm{HV}$ ) and a 4 hydroxyacid (4HV) by supplying $3 \mathrm{HB}$ from glucose through PhaAB reactions and $3 \mathrm{HV}$ and $4 \mathrm{HV}$ from LA through LvaEDABC reactions.

After $96 \mathrm{~h}$ of cultivation, a $\mathrm{P}(3 \mathrm{HB}-c o-3 \mathrm{HV}-c o-4 \mathrm{HV})$ terpolymer with $1.4 \mathrm{~g} / \mathrm{l} \mathrm{DCW}$ and $35.6 \mathrm{wt} \%$ and a monomer molar ratio of 88:6:6 were obtained from the PHBV11 strain. The content of 3HB monomer increased from $85.3 \mathrm{~mol} \%$ in the terpolymer compared to $87.9 \mathrm{~mol} \%$ in the PHBV produced by the PHV 11 strain. However, the amount of total HV monomer was still $12.1 \mathrm{~mol} \%$. The DCW also reduced slightly from $1.6 \mathrm{~g} / \mathrm{l}$ to $1.4 \mathrm{~g} / \mathrm{l}$ (Table 2, Fig. 3). In comparison with $P$. putida KT2440 strains that produced PHBV with 9.4 mol\% 3HB, 67.1 $\mathrm{mol} \% 3 \mathrm{HV}$, and $23.5 \mathrm{~mol} \% 4 \mathrm{HV}$ [18], the E. coli strain produced PHBV with a higher proportion of the $3 \mathrm{HB}$ monomer.

The observed variation in terpolymer monomer ratio might be attributed to differences in $\mathrm{PhaC}_{\mathrm{Cv}}$ expression, $3 \mathrm{HB}$ monomer availability under different $\mathrm{PhaAB}$ expression patterns, and variation in the cellular physiology of host cells E. coli MG1655 and P. putida KT2440.

In conclusion, E. coli provides a well-established culture strategy for achieving high cell densities and fragility of 


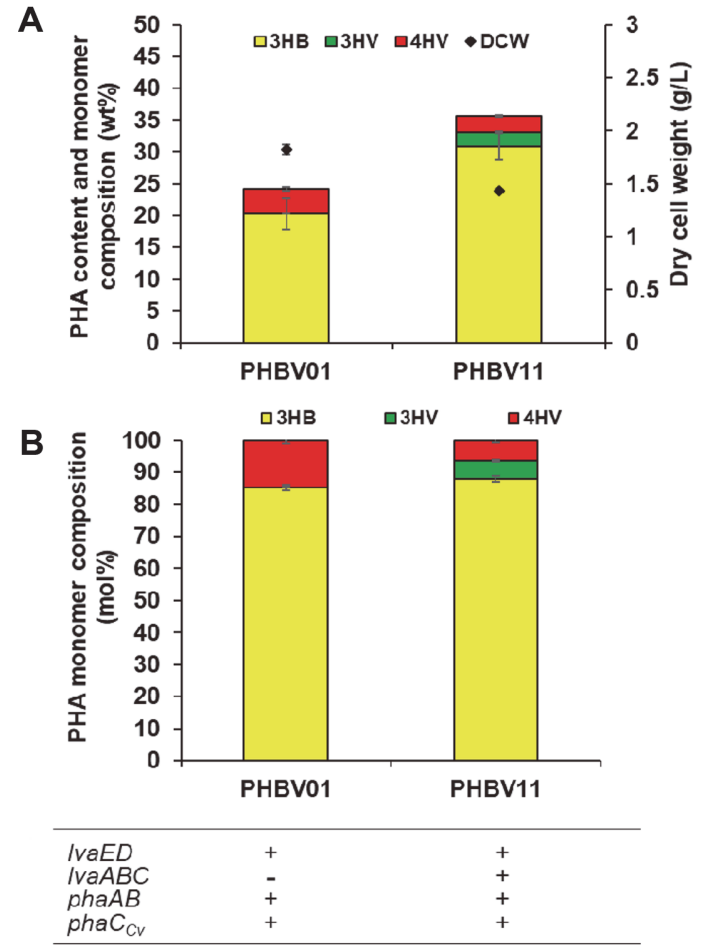

Fig. 3. Cellular PHA content (wt\%), dry cell weight (g/l) (A) and PHA monomer composition (mol\%) (B) in PHBV01 harboring pBbB6a-lvaED and pBbA2c-phaCAB, and PHBV11 harboring pBbB6a-lvaED, pBbE6k-lvaABC and pBbA2c-phaCAB. Samples were taken and analyzed after $96 \mathrm{~h}$ of cultivation. Yellow, $3 \mathrm{HB}$; red, $4 \mathrm{HV}$; green, $3 \mathrm{HV}$. The table indicates the enzymes overexpressed from plasmids. Error bars indicate standard deviations of three independent cultivations.

cells, facilitating the simple isolation and purification of biopolymers [20,40]. Four distinct varieties of PHAs, namely $\mathrm{p}(4 \mathrm{HV}), \mathrm{p}(3 \mathrm{HV}-c o-4 \mathrm{HV}), \mathrm{p}(3 \mathrm{HB}-c o-4 \mathrm{HV})$, and $\mathrm{p}(3 \mathrm{HB}-c o-3 \mathrm{HV}-c o-4 \mathrm{HV})$ were produced using metabolically engineered E. coli. Although E. coli produces less PHA than P. putida KT2440, the results from this study demonstrate that it can serve as a host to produce PHAs with HV monomers synthesized from LA after further metabolic engineering. This research also provides an alternative approach for effectively supplying HV monomers from a cost-effective alternative for PHA synthesis rather than using costly substrates like GVL, valeric acid, or propionate.

\section{Acknowledgments}

This work has supported by the National Research Foundation of Korea (NRF) grant funded by the Korea government (MIST) (2020R1A4A1018332) and granted by innovative science project in 2020 of The circle foundation

\section{Conflict of Interest}

The authors have no financial conflicts of interest to declare.

\section{References}

1. Reinecke F, Steinbüchel A. 2009. Ralstonia eutropha strain H16 as model organism for PHA metabolism and for biotechnological production of technically interesting biopolymers. Microb. Physiol. 16: 91-108.

2. Yu J. 2007. Microbial production of bioplastics from renewable resources, pp. 585-610. Bioprocessing for value-added products from renewable resources, Ed. Elsevier,

3. Pettinari MJ, Vázquez GJ, Silberschmidt D, Rehm B, Steinbüchel A, Méndez BS. 2001. Poly (3-hydroxybutyrate) synthesis genes in Azotobacter sp. strain FA8. Appl. Environ. Microbiol. 67: 5331-5334.

4. Lütke-Eversloh T, Steinbüchel A. 2004. Microbial polythioesters. Macromol. Biosci. 4: 165-174.

5. Napper IE, Thompson RC. 2019. Environmental deterioration of biodegradable, oxo-biodegradable, compostable, and conventional plastic carrier bags in the sea, soil, and open-air over a 3-year period. Environ. Sci. Technol. 53: 4775-4783.

6. Bhatia SK, Gurav R, Choi T-R, Jung H-R, Yang S-Y, Moon Y-M, et al. 2019. Bioconversion of plant biomass hydrolysate into bioplastic (polyhydroxyalkanoates) using Ralstonia eutropha 5119. Bioresour. Technol. 271: 306-315.

7. Lemoigne M. 1926. Products of dehydration and of polymerization of $\beta$-hydroxybutyric acid. Bull. Chem. Soc. Japan 8: $770-782$.

8. Muzaiyanah AR, Amirul AA. 2013. Studies on the microbial synthesis and characterization of polyhydroxyalkanoates containing 4hydroxyvalerate using $\gamma$-valerolactone. Appl. Biochem. Biotechnol. 170: 1194-1215. 
9. Raza ZA, Abid S, Banat IM. 2018. Polyhydroxyalkanoates: Characteristics, production, recent developments and applications. Int. Biodeterior. Biodegradation 126: 45-56.

10. Możejko-Ciesielska J, Kiewisz R. 2016. Bacterial polyhydroxyalkanoates: Still fabulous? Microbiol. Res. 192: 271-282.

11. Sheu D-S, Chen Y-LL, Jhuang W-J, Chen H-Y, Jane W-N. 2018. Cultivation temperature modulated the monomer composition and polymer properties of polyhydroxyalkanoate synthesized by Cupriavidus sp. L7L from levulinate as sole carbon source. Int. J. Biol. Macromol. 118: 1558-1564.

12. Gahlawat G, Soni SK. 2017. Valorization of waste glycerol for the production of poly (3-hydroxybutyrate) and poly (3hydroxybutyrate-co-3-hydroxyvalerate) copolymer by Cupriavidus necator and extraction in a sustainable manner. Bioresour. Technol. 243: 492-501.

13. Schmack G, Gorenflo V, Steinbüchel A. 1998. Biotechnological production and characterization of polyesters containing 4hydroxyvaleric acid and medium-chain-length hydroxyalkanoic acids. Macromolecules 31: 644-649.

14. Lee W-H, Loo C-Y, Nomura CT, Sudesh K. 2008. Biosynthesis of polyhydroxyalkanoate copolymers from mixtures of plant oils and 3-hydroxyvalerate precursors. Bioresour. Technol. 99: 6844-6851.

15. Valentin HE, Steinbüchel A. 1995. Accumulation of poly(3-hydroxybutyric acid-co-3-hydroxyvaleric acid-co-4-hydroxyvaleric acid) by mutants and recombinant strains of Alcaligenes eutrophus. J. Environ. Polymer Degradation 3: 169-175.

16. Koller M, Hesse P, Fasl H, Stelzer F, Braunegg G. 2017. Study on the effect of levulinic acid on whey-based biosynthesis of Poly(3hydroxybutyrate-co-3-hydroxyvalerate) by Hydrogenophaga pseudoflava. Appl. Food Biotechnol. 4: 65-78.

17. Novackova I, Kucera D, Porizka J, Pernicova I, Sedlacek P, Koller M, et al. 2019. Adaptation of Cupriavidus necator to levulinic acid for enhanced production of $\mathrm{P}(3 \mathrm{HB}-\mathrm{co}-3 \mathrm{HV})$ copolyesters. Biochem. Eng. J. 151: 107350.

18. Cha D, Ha HS, Lee SK. 2020. Metabolic engineering of Pseudomonas putida for the production of various types of short-chain-length polyhydroxyalkanoates from levulinic acid. Bioresour. Technol. 309: 123332

19. Bozell JJ, Moens L, Elliott DC, Wang Y, Neuenscwander GG, Fitzpatrick SW, et al. 2000. Production of levulinic acid and use as a platform chemical for derived products. Resour. Conserv. Recycl. 28: 227-239.

20. Favaro L, Basaglia M, Casella S. 2019. Improving polyhydroxyalkanoate production from inexpensive carbon sources by genetic approaches: a review. Biofuels Bioprod. Biorefining 13: 208-227.

21. Kidwell J, Valentin HE, Dennis D. 1995. Regulated expression of the Alcaligenes eutrophus pha biosynthesis genes in Escherichia coli. Appl. Environ. Microbiol. 61: 1391-1398.

22. Langenbach S, Rehm BHA, Steinbüchel A. 1997. Functional expression of the PHA synthase gene phaC1 from Pseudomonas aeruginosa in Escherichia coli results in poly(3-hydroxyalkanoate) synthesis. FEMS Microbiol. Lett. 150: 303-309.

23. Ren Q, Sierro N, Kellerhals M, Kessler B, Witholt B. 2000. Properties of engineered poly-3-hydroxyalkanoates produced in recombinant Escherichia coli strains. Appl. Environ. Microbiol. 66: 1311-1320.

24. Ren Q, Beilen JBv, Sierro N, Zinn M, Kessler B, Witholt B. 2005. Expression of PHA polymerase genes of Pseudomonas putida in Escherichia coli and its effect on PHA formation. Antonie Van Leeuwenhoek 87: 91-100.

25. Wang Q, Yu H, Xia Y, Kang Z, Qi Q. 2009. Complete PHB mobilization in Escherichia coli enhances the stress tolerance: a potential biotechnological application. Microb. Cell Fact. 8: 1-9.

26. Rand JM, Pisithkul T, Clark RL, Thiede JM, Mehrer CR, Agnew DE, et al. 2017. A metabolic pathway for catabolizing levulinic acid in bacteria. Nat. Microbiol. 2: 1624-1634.

27. Lee TS, Krupa RA, Zhang F, Hajimorad M, Holtz WJ, Prasad N, et al. 2011. BglBrick vectors and datasheets: A synthetic biology platform for gene expression. J. Biol. Eng. 5: 12.

28. Gibson DG, Young L, Chuang R-Y, Venter JC, Hutchison CA, Smith HO. 2009. Enzymatic assembly of DNA molecules up to several hundred kilobases. Nat. Methods 6: 343-345

29. Reis AC, Salis HM. 2020. An automated model test system for systematic development and improvement of gene expression models. ACS Synth. Biol. 9: 3145-3156.

30. Gorenflo V, Schmack G, Vogel R, Steinbüchel A. 2001. Development of a process for the biotechnological large-scale production of 4hydroxyvalerate-containing polyesters and characterization of their physical and mechanical properties. Biomacromolecules 2: 45-57.

31. Juengert JR, Bresan S, Jendrossek D. 2018. Determination of polyhydroxybutyrate (PHB) content in Ralstonia eutropha using gas chromatography and nile red staining. Bio Protoc. 8: e2748.

32. Peña C, Castillo T, García A, Millán M, Segura D. 2014. Biotechnological strategies to improve production of microbial poly-(3-hydroxybutyrate): a review of recent research work. Microb. Biotechnol. 7: 278-293.

33. Matsumoto Ki, Yamada M, Leong CR, Jo S-J, Kuzuyama T, Taguchi S. 2011. A new pathway for poly (3-hydroxybutyrate) production in Escherichia coli and Corynebacterium glutamicum by functional expression of a new acetoacetyl-coenzyme A synthase. Biosci. Biotechnol. Biochem. 75: 364-366.

34. Snell KD, Feng F, Zhong L, Martin D, Madison LL. 2002. YfcX enables medium-chain-length poly (3-hydroxyalkanoate) formation from fatty acids in recombinant Escherichia coli fadB strains. J. Bacteriol. 184: 5696-5705.

35. Liu S-J, Steinbüchel A. 2000. A novel genetically engineered pathway for synthesis of poly(hydroxyalkanoic acids) in Escherichia coli. Appl. Environ. Microbiol. 66: 739-743.

36. Kim BS, Lee SY. 2000. Production of poly (3-hydroxybutyrate) from inexpensive substrates. Enzyme Microb. Technol. 27 : 774-777.

37. Kim D, Sathesh-Prabu C, JooYeon Y, Lee SK. 2019. High-level production of 4-hydroxyvalerate from levulinic acid via whole-cell biotransformation decoupled from cell metabolism. J. Agric. Food Chem. 67: 10678-10684.

38. Steinbüchel A, Debzi E-M, Marchessault RH, Timm A. 1993. Synthesis and production of poly (3-hydroxyvaleric acid) homopolyester by Chromobacterium violaceum. Appl. Microbiol. Biotechnol. 39: 443-449.

39. Tripathi L, Wu L-P, Dechuan M, Chen J, Wu Q, Chen G-Q. 2013. Pseudomonas putida KT2442 as a platform for the biosynthesis of polyhydroxyalkanoates with adjustable monomer contents and compositions. Bioresour. Technol. 142: 225-231.

40. Aldor IS, Keasling JD. 2003. Process design for microbial plastic factories: metabolic engineering of polyhydroxyalkanoates. Curr. Opin. Biotechnol. 14: 475-483.

41. Blattner FR, Plunkett G, Bloch CA, Perna NT, Burland V, Riley M, et al. 1997. The complete genome sequence of Escherichia coli K12. Science 277: 1453-1462

42. Durfee T, Nelson R, Baldwin S, Plunkett G, Burland V, Mau B, et al. 2008. The complete genome sequence of Escherichia coli DH10b: insights into the biology of a laboratory workhorse. J. Bacteriol. 190: 2597-2606. 